\title{
Survivin and XIAP expression in distinct tumor compartments of surgically resected gastric cancer: XIAP as a prognostic marker in diffuse and mixed type adenocarcinomas
}

\author{
LEVENT DIZDAR ${ }^{1}$, MONIKA TOMCZAK ${ }^{1}$, THOMAS A. WERNER ${ }^{1}$, SAMI A. SAFI $^{1}$, JASMIN C. RIEMER $^{2}$, \\ PABLO E. VERDE ${ }^{3}$, NIKOLAS H. STOECKLEIN ${ }^{1}$, WOLFRAM T. KNOEFEL ${ }^{1}$ and ANDREAS KRIEG ${ }^{1}$ \\ ${ }^{1}$ Department of Surgery (A); ${ }^{2}$ Institute of Pathology; ${ }^{3}$ Coordination Centre for Clinical Trials, \\ Heinrich-Heine-University and University Hospital Duesseldorf, D-40225 Duesseldorf, Germany
}

Received March 1, 2017; Accepted August 3, 2017

DOI: $10.3892 / 01.2017 .6999$

\begin{abstract}
There is considerable evidence that the inhibitor of apoptosis protein (IAP) family serves a role in tumorigenesis. The most studied IAP family members, survivin and X-linked inhibitor of apoptosis (XIAP), have been demonstrated to serve as biomarkers in distinct tumor entities. Thus, the present study aimed to investigate the expression levels of both IAPs in the tumor center, invasion front and lymph node metastases of surgically resected gastric cancer (GC) specimens. Tissue microarrays containing samples from 201 primary GCs were analyzed. IAP expression was detected using immunohistochemistry in different tumor compartments, normal mucosa and lymph node metastases. In addition, the association between the expression levels of these proteins, and clinicopathological parameters and overall survival was investigated. High levels of survivin and XIAP were evident in GC, when compared with normal mucosa, and were correlated with intestinal-type and well-differentiated GC, as well as low International Union Against Cancer stages. Increased XIAP expression was detected in lymph node metastases as compared with corresponding primary tumors. XIAP overexpression was identified to be an independent negative prognostic marker in diffuse and mixed type GC. These results suggest a potential role of survivin and XIAP in the early phase of gastric carcinogenesis. In addition, increased XIAP expression in lymph node metastases supports the observation that IAPs serve an essential role in metastatic tumor disease. Since XIAP expression was identified to be associated with poor survival in diffuse and mixed type GC, XIAP may serve as a novel therapeutic target in these types of GC.
\end{abstract}

Correspondence to: Dr Andreas Krieg, Department of Surgery (A), Heinrich-Heine-University and University Hospital Duesseldorf, Moorenstreet 5, Building 12.46, D-40225 Duesseldorf, Germany

E-mail: andreas.krieg@med.uni-duesseldorf.de

Key words: survivin, XIAP, gastric cancer, prognostic marker

\section{Introduction}

Despite its steadily declining incidence, gastric cancer (GC) causes more than 723,000 deaths a year and is still the third-leading cause of cancer-related death worldwide (1). Poor survival of GC is attributed to the high percentage of patients with an advanced stage of the disease at the time of first diagnosis. Approximately 35\% of GC patients harbor distant metastasis at first clinical presentation (2). In addition, GC is characterized by a high rate of locoregional as well as distant recurrence after surgical resection with curative intend (3). Currently, the basis for treatment of patients with metastatic or recurrent disease is chemotherapy. Although some of these regimens demonstrated activity in recent phase III trials the median overall survival of patients with advanced GC remains disappointing (4-7). To improve the outcome of patients with advanced GC novel treatment options are urgently needed. One promising approach might be the use of targeted anticancer therapies. Such therapeutic regimes aim to inhibit cancer development by interfering with distinct molecules which are involved in pathways that regulate important cancer cell abilities like resistance to growth inhibition and apoptosis, cell invasion, metastasis or the induction of angiogenesis (8). One common principle to identify such target molecules is to study the expression and prognostic significance of proteins in cancer tissue specimens.

In this context, the inhibitor of apoptosis protein (IAP) family has attracted considerable attention. IAPs significantly influence essential biological functions during cancer development as well as resistance to conventional chemotherapeutic agents by inhibiting cell death pathways and by promoting cancer cell proliferation, migration and metastasis at the same time (9-12). The most extensively studied IAP family members are survivin/BIRC5 and X-linked inhibitor of apoptosis (XIAP/BIRC4) $(13,14)$. Undoubtedly, both survivin and XIAP are multifunctional proteins regulating distinct cellular processes $(9,11,13,15-18)$. However, survivin and XIAP have been demonstrated to unfold their antiapoptotic and pro-metastatic activities by direct interaction. In this context, survivin has been implicated in stabilization of XIAP thus preventing XIAP from polyubiquitination and 
subsequent proteosomal degradation (19). Consequently, a survivin-XIAP complex synergistically inhibits caspases and activates NF- $\kappa \mathrm{B}$ signaling $(11,20)$. Of note, $\mathrm{NF}-\kappa \mathrm{B}$ activation by a survivin-XIAP complex was linked to tumor cell invasion and formation of metastasis (11). Although the expression of survivin and XIAP could be correlated with clinicopathological parameters and was linked to poor survival in GC (21-27), none of these studies took the pro-invasive and metastatic functions of a survivin-XIAP complex into account and analyzed the expression of both IAPs in different tumor compartments such as the tumor center, invasion front and metastases. Thus, aim of our study was to specifically investigate the expression of survivin and XIAP in the tumor center, invasion front and lymph node metastases of surgically resected specimens of GC. Considering the synergistic function of XIAP and survivin, we assessed the prognostic value of the tumor compartment-related expression of both IAPs and its association with clinicopathological variables in GC patients according to the REporting recommendations for tumor MARKer prognostic studies (REMARK) (28).

\section{Materials and methods}

Patient selection and clinicopathological data. Medical charts of patients with primary gastric adenocarcinoma who had undergone surgical resection at the Department of Surgery (A) of the University Hospital Duesseldorf (Duesseldorf, Germany) between 1995 and 2013 were retrospectively reviewed. All patients were treated in curative intend, using total or subtotal gastrectomy and D2 lymphadenectomy. Unfortunately, for most of the patients information on adjuvant chemotherapy was unavailable. Exclusion criteria for this study were preoperative chemotherapy, incomplete pathological report and insufficient tumor material for further analysis. Patients lost to follow up, or with incomplete resection and patients who died because of postoperative complications within 30 days after surgery were excluded from our survival analysis. The study was carried out in accordance to Good Clinical Practice, the Declaration of Helsinki and local rules as well as regulations of the country. The Institutional Review Board (IRB) of the Medical Faculty, Heinrich Heine University Duesseldorf approved the use of tissue specimens and clinicopathological data for this study (IRB-no. 3821). Clinicopathological parameters were extracted from the clinical and histopathological reports. Surgically resected tumors were staged according to the 7 th TNM edition of the UICC (29). Overall survival data were retrieved from a prospectively maintained database at the Department of Surgery (A).

Tissue microarray and immunohistochemistry. Formalin-fixed and paraffin embedded (FFPE) tumor specimens were obtained from the Institute of Pathology, University Hospital Duesseldorf. Tissue microarray (TMA) construction was performed as recently described (30). If available two cylinders of $1.0 \mathrm{~mm}$ diameter were taken from the tumor center and invasion front, one from tumor surrounding non-malignant mucosa and one from a lymph node metastasis. As control for immunohistochemical reaction two cylinders of normal tonsille tissue and colon cancer were inserted into the right upper corner of each recipient block. For immunohistochemistry $2 \mu \mathrm{m}$ sections were cut from each TMA block and mounted on superfrost glass slides.

For immunohistochemical staining procedures rabbit polyclonal anti-survivin antibody (NB500-201; 1:1,000 dilution; Novus Biologicals, Littleton, $\mathrm{CO}$, USA) and mouse monoclonal anti-XIAP antibody (Clone 48; 1:50 dilution; BD Biosciences, San Jose, CA, USA) were used. Isotype control was conducted using mouse IgG1 kappa (MOPC-21; Abcam, Cambridge, UK) and rabbit Immunoglobulin Fraction (Code X0903; Dako, Glostrup, Denmark). Expression levels were estimated according to an immunoreactivity score (IRS) as described previously (30-32).

Two independent investigators who were blinded to clinicopathological data examined all sections (LD and MT). In case of differing results both observers re-examined the slide simultaneously and a consensus decision was made. For survivin, nuclear and cytoplasmic protein expression were separately determined.

Statistical analysis. The mean IRS of both tissue cores from tumor centers and invasion fronts was calculated for statistical analysis. Variation of IAP expression in different tumor compartments was analyzed using the Mann-Whitney U test or Wilcoxon matched pairs test, as indicated. Spearman's correlation coefficient was used to test a relationship between survivin and XIAP expression. For survival analysis and to compare expression levels according to clinicopathological variables, the median IRS for nuclear and cytoplasmic survivin as well as for XIAP was combined and categorized into groups of high ( $>$ median IRS $=$ IRS $>2)$ or low $(\leq$ median IRS =IRS $\leq 2$ ) survivin and XIAP expression. This homogenous cut-off value divided our collective of GC patients into two approximately equally sized groups for both IAPs, survivin and XIAP. Categorical data were examined using the Fischer's exact test. Overall survival was defined as the period from the date of surgery until the date of last follow up or until death of any cause. For univariate survival analysis, Kaplan-Meier curves were generated and assessed using the log-rank (Mantel Cox) test. In addition, hazard ratios (HR) with $95 \%$ confidence intervals (CI) were estimated and multiple regression analysis was performed with the proportional hazard Cox's regression. Akaike Information Criterion (AIC) was applied for variable selection, the model with the lowest AIC was considered as the model which better fits the data (33). Statistical analyses were performed using GraphPad Prism for Windows (Version 5, GraphPad Software, San Diego, CA, USA), SPSS statistics for Windows (Version 17.0; SPSS, Inc., Chicago, IL, USA) and the R statistical software, version 3.2.2. $\mathrm{P}<0.05$ was considered to indicate a statistically significant difference.

\section{Results}

Patients and outcome. Using our pre-defined inclusion and exclusion criteria, a total number of 201 patients who underwent total or subtotal gastrectomy with D2 lymphadenectomy for GC between 1995 and 2013 could be included in our study. Patient's pathological and clinical characteristics are summarized in Table I. Only 154 patients with a median follow-up of 
Table I. Patient characteristics $(n=201)$.

Variable

No. of patients $(\%)$

Total 201

Age

Median (range); years

$71(26-91)$

Gender

Male

Female

Lauren classification

Intestinal type

Diffuse type

Mixed type

Tumor stage

$\mathrm{T} 1 / 2$

$\mathrm{T} 3 / 4$

Lymph node metastasis

N0

$\mathrm{N} 1+$

Distant metastasis

M0

M1

Grading

G1/2

G3/4

UICC stage

UICC I/II

UICC III/IV

Resection status

R0

$\mathrm{R}+$
25.3 month (range 1-120 month) and median overall survival of 29.8 (range 1-120) months could be included into our survival analysis, as 24 patients with incomplete resection, 14 patients who died within 30 days after surgery and 9 patients who were lost to follow-up had to be excluded. 103 (66.9\%) patients died during the follow-up period.

Survivin and XIAP expression in GC. Immunohistochemical staining of TMAs revealed a cytoplasmic and nuclear expression of survivin, whereas XIAP expression was exclusively detected in the cytoplasm (Fig. 1A). First, we analyzed expression patterns of survivin and XIAP in different GC compartments (tumor center, invasion front and lymph node metastasis) and corresponding normal mucosa. As shown in Fig. 1B survivin and XIAP expression levels were significantly elevated in GC tissues when compared to non-neoplastic mucosa. Furthermore, an increased expression of XIAP in lymph node metastases became evident. However, there was no difference in survivin and XIAP expression levels when comparing tumor centers with tumor invasion fronts (Fig. 1C). Of note, cytoplasmic survivin

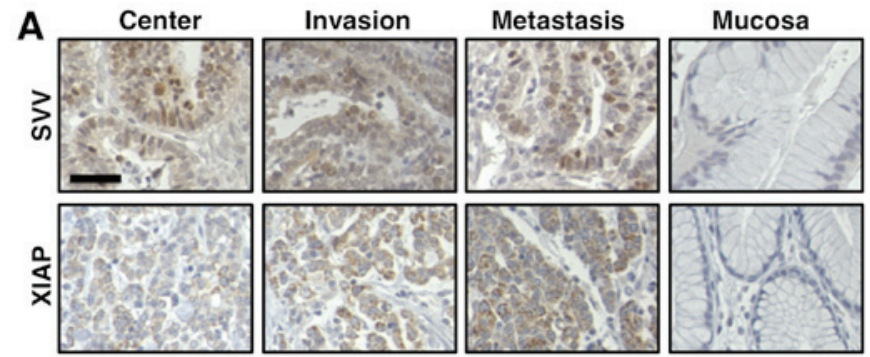

B
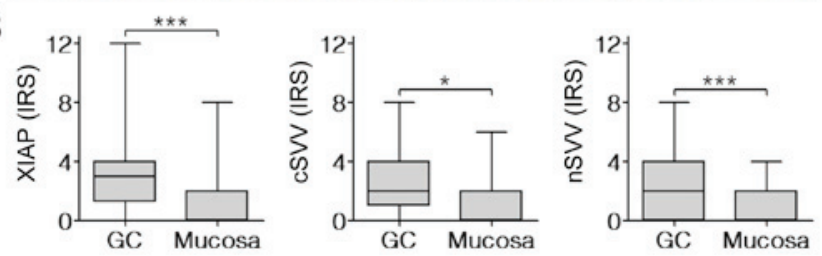

C
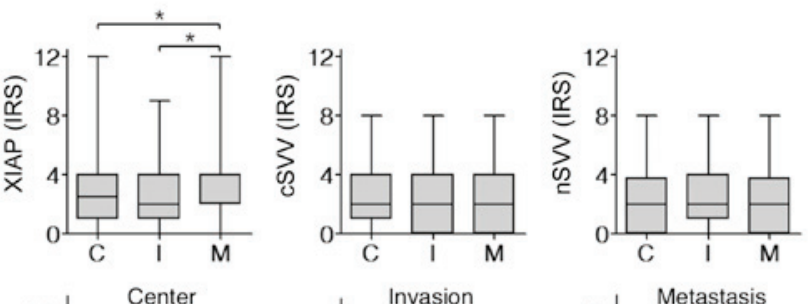

D
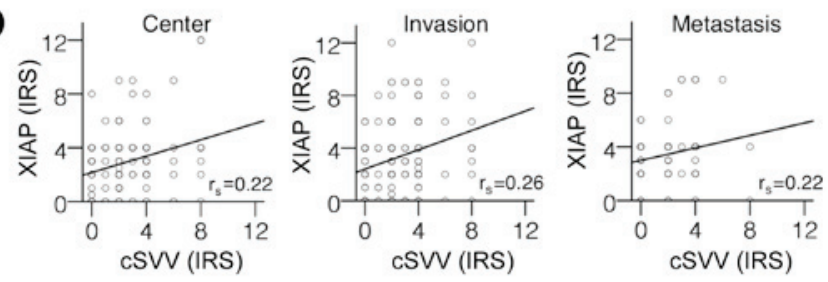

Figure 1. (A) Representative images of immunohistochemical staining for survivin (SVV) (top) and X-linked inhibitor of apoptosis (XIAP) (bottom) in GC tissue samples from the tumor center [Center (C)], invasion front [Invasion (I)], lymph node metastasis [metastasis (M)] as well as adjacent non-malignant mucosa (Mucosa) from one patient. Images were captured at x200 magnification and scale bar indicates $25 \mu \mathrm{m}$. (B) XIAP, cytoplasmic SVV (cSVV) and nuclear SVV (nSVV) expression were significantly increased in GC tissue, when compared to non-malignant mucosa. Data were analyzed using the two-tailed nonparametric Mann-Whitney $\mathrm{U}$ test $\left({ }^{*} \mathrm{P}<0.05\right.$; $\left.{ }^{* * * *} \mathrm{P}<0.001\right)$. (C) Boxplots display the expression of XIAP and SVV in tumor centers (C), tumor invasion fronts (I) and lymph node metastasis (M) of corresponding GC tissue samples. Data were analyzed by Wilcoxon matched pairs test $\left({ }^{*} \mathrm{P}<0.05\right)$. (D) XIAP and cSVV expression were positively correlated in different GC compartments (Center: rs=0.219, $\mathrm{P}=0.009$; Invasion: $\mathrm{rs}=0.257, \mathrm{P}=0.001$; lymph node metastasis; Metastasis: $\mathrm{rs}=0.218, \mathrm{P}=0.047$ ).

expression correlated positively with XIAP expression in distinct tumor compartments (Center: $\mathrm{P}=0.009$; Invasion front: $\mathrm{P}=0.001$; Lymph node metastasis: $\mathrm{P}=0.047$; Fig. 1D).

Correlation between survivin or XIAP and clinicopathological factors. Next, we categorized the respective IRS of survivin and XIAP in primary tumors into high (IRS >2) and low (IRS $\leq 2$ ) expression and correlated these data with clinicopathological parameters (Tables II-IV). Using this categorization, we found that except for nuclear survivin in tumor centers, both IAPs exhibited higher expression levels in intestinal type GCs when compared to the diffuse type. In addition, well or moderately differentiated GC specimens demonstrated higher XIAP expression in both primary tumor compartments and increased cytoplasmic survivin in the tumor center. Interestingly, high survivin and XIAP 
Table II. Correlation between XIAP expression and clinicopathological factors in gastric cancer.

\begin{tabular}{|c|c|c|c|c|c|c|c|c|c|c|}
\hline & \multicolumn{5}{|c|}{ XIAP-center } & \multicolumn{5}{|c|}{ XIAP-invasion } \\
\hline & \multicolumn{2}{|c|}{ Low $n=67$} & \multicolumn{2}{|c|}{ High $n=82$} & \multirow[b]{2}{*}{ P-value } & \multicolumn{2}{|c|}{ Low $n=88$} & \multicolumn{2}{|c|}{ High n=94 } & \multirow[b]{2}{*}{ P-value } \\
\hline & $\mathrm{n}$ & $\%$ & $\mathrm{n}$ & $\%$ & & $\mathrm{n}$ & $\%$ & $\mathrm{n}$ & $\%$ & \\
\hline Age & & & & & 0.514 & & & & & 0.302 \\
\hline$\leq$ Median & 33 & 42.3 & 45 & 57.7 & & 50 & 52.1 & 46 & 47.9 & \\
\hline$>$ Median & 34 & 47.9 & 37 & 52.1 & & 38 & 44.2 & 48 & 55.8 & \\
\hline Sex & & & & & 0.369 & & & & & 0.429 \\
\hline Men & 17 & 38.6 & 27 & 61.4 & & 25 & 43.9 & 32 & 56.1 & \\
\hline Women & 50 & 47.6 & 55 & 52.4 & & 63 & 50.4 & 62 & 49.6 & \\
\hline Lauren & & & & & $<0.001$ & & & & & 0.005 \\
\hline diffuse & 34 & 59.6 & 23 & 40.4 & & 38 & 57.6 & 28 & 42.4 & \\
\hline intestinal & 15 & 25.4 & 44 & 74.6 & & 24 & 33.3 & 48 & 66.7 & \\
\hline mixed & 18 & 54.5 & 15 & 45.5 & & 26 & 59.1 & 18 & 40.9 & \\
\hline Tumor stage & & & & & 0.014 & & & & & 0.049 \\
\hline $\mathrm{T} 1 / \mathrm{T} 2$ & 28 & 35.4 & 51 & 64.6 & & 47 & 42.3 & 64 & 57.7 & \\
\hline $\mathrm{T} 3 / \mathrm{T} 4$ & 39 & 55.7 & 31 & 44.3 & & 41 & 57.7 & 30 & 42.3 & \\
\hline Lymph nodes & & & & & 0.074 & & & & & 0.098 \\
\hline Negative. N0 & 10 & 30.3 & 23 & 69.7 & & 19 & 38.0 & 31 & 62.0 & \\
\hline Positive. N+ & 57 & 49.1 & 59 & 50.9 & & 69 & 52.3 & 63 & 47.7 & \\
\hline Metastasis & & & & & 0.119 & & & & & 0.448 \\
\hline M0 & 56 & 42.4 & 76 & 57.6 & & 78 & 47.3 & 87 & 52.7 & \\
\hline M1 & 11 & 64.7 & 6 & 35.3 & & 10 & 58.8 & 7 & 41.2 & \\
\hline Grading & & & & & 0.011 & & & & & 0.023 \\
\hline G1/G2 & 9 & 25.7 & 26 & 74.3 & & 14 & 32.6 & 29 & 67.4 & \\
\hline G3/G4 & 58 & 50.9 & 56 & 49.1 & & 74 & 53.2 & 65 & 46.8 & \\
\hline Resection margins & & & & & 0.075 & & & & & 1.000 \\
\hline Negative. R0 & 55 & 42.0 & 76 & 58.0 & & 79 & 48.5 & 84 & 51.5 & \\
\hline Positive. R1 & 12 & 66.7 & 6 & 33.3 & & 9 & 47.4 & 10 & 52.6 & \\
\hline UICC & & & & & 0.019 & & & & & 0.012 \\
\hline $\mathrm{I} / \mathrm{II}$ & 20 & 32.8 & 41 & 67.2 & & 33 & 38.4 & 53 & 61.6 & \\
\hline III/IV & 47 & 53.4 & 41 & 46.6 & & 55 & 57.3 & 41 & 42.7 & \\
\hline
\end{tabular}

Bold print denotes $\mathrm{P}<0.05$. XIAP, $\mathrm{X}$-linked inhibitor of apoptosis.

expression in distinct tumor compartments was associated with less advanced UICC stages and tumors with an invasion restricted to the muscularis propria. Surprisingly, tumors without lymph node metastasis showed higher cytoplasmic survivin expression levels in the invasion front.

XIAP is an independent negative prognostic marker in diffuse and mixed type GC. To explore the prognostic value of survivin and XIAP in GC, we performed survival analysis including all GCs, irrespective of their histological type. Accordingly, univariate survival analysis revealed that high tumor stage, lymph node metastasis, presence of distant metastasis, poor differentiation $(\mathrm{G} 3+4)$, advanced UICC stages and low cytoplasmic survivin expression in the tumor invasion front were significantly associated with a poor outcome (Table V, Fig. 2A). However, multivariate analysis determined only advanced
UICC stages, G3/G4 differentiation and male gender as independent predictors of survival in our study cohort (Table VI).

The observation that survivin and XIAP expression are associated with different histologic types, prompted us to perform a survival analysis for each histologic subtype. Thus, univariate survival analysis of intestinal type GCs demonstrated that high tumor stage, lymph node metastasis, poorly differentiated tumors, advanced UICC stage and low XIAP expression levels in tumor centers were related to worse overall survival (Table V, Fig. 2B). In contrast, univariate analysis of the diffuse type revealed, that increased XIAP expression in tumor centers, patient's age, T3/4 tumors and advanced UICC stages as well as distant metastasis were significantly associated with a poor prognosis (Table V, Fig. 2C). In mixed type GCs T3/4 stage, UICC III/IV stages and high XIAP expression in tumor centers were of prognostic relevance 
Table III. Correlation between cytoplasmic SVV expression and clinicopathological factors in gastric cancer.

\begin{tabular}{|c|c|c|c|c|c|c|c|c|c|c|}
\hline & \multicolumn{5}{|c|}{ SVV (cyt.)-center } & \multicolumn{5}{|c|}{ SVV (cyt.)-invasion } \\
\hline & \multicolumn{2}{|c|}{ Low $n=92$} & \multicolumn{2}{|c|}{ High $n=56$} & \multirow[b]{2}{*}{ P-value } & \multicolumn{2}{|c|}{ Low $n=100$} & \multicolumn{2}{|c|}{ High $n=74$} & \multirow[b]{2}{*}{ P-value } \\
\hline & $\mathrm{n}$ & $\%$ & $\mathrm{n}$ & $\%$ & & $\mathrm{n}$ & $\%$ & $\mathrm{n}$ & $\%$ & \\
\hline Age & & & & & 0.866 & & & & & 0.221 \\
\hline$\leq$ Median & 48 & 63.2 & 28 & 36.8 & & 56 & 62.2 & 34 & 37.8 & \\
\hline$>$ Median & 44 & 61.1 & 28 & 38.9 & & 44 & 52.4 & 40 & 47.6 & \\
\hline Sex & & & & & 0.256 & & & & & 0.068 \\
\hline Men & 29 & 70.7 & 12 & 29.3 & & 37 & 68.5 & 17 & 31.5 & \\
\hline Women & 63 & 58.9 & 44 & 41.1 & & 63 & 52.5 & 57 & 47.5 & \\
\hline Lauren & & & & & $<0.001$ & & & & & $<0.001$ \\
\hline diffuse & 48 & 87.3 & 7 & 12.7 & & 50 & 79.4 & 13 & 20.6 & \\
\hline intestinal & 26 & 43.3 & 34 & 56.7 & & 33 & 46.5 & 38 & 53.5 & \\
\hline mixed & 18 & 54.5 & 15 & 45.5 & & 17 & 42.5 & 23 & 57.5 & \\
\hline Tumor stage & & & & & 0.501 & & & & & 0.041 \\
\hline $\mathrm{T} 1 / \mathrm{T} 2$ & 47 & 59.5 & 32 & 40.5 & & 54 & 50.9 & 52 & 49.1 & \\
\hline $\mathrm{T} 3 / \mathrm{T} 4$ & 45 & 65.2 & 24 & 34.8 & & 46 & 67.6 & 22 & 32.4 & \\
\hline Lymph nodes & & & & & 0.690 & & & & & 0.007 \\
\hline Negative. N0 & 20 & 58.8 & 14 & 41.2 & & 20 & 40.8 & 29 & 59.2 & \\
\hline Positive. N+ & 72 & 63.2 & 42 & 36.8 & & 80 & 64.0 & 45 & 36.0 & \\
\hline Metastasis & & & & & 0.597 & & & & & 1.000 \\
\hline M0 & 80 & 61.1 & 51 & 38.9 & & 91 & 57.6 & 67 & 42.4 & \\
\hline M1 & 12 & 70.6 & 5 & 29.4 & & 9 & 56.3 & 7 & 43.8 & \\
\hline Grading & & & & & 0.003 & & & & & 0.051 \\
\hline $\mathrm{G} 1 / \mathrm{G} 2$ & 14 & 40.0 & 21 & 60.0 & & 19 & 44.2 & 24 & 55.8 & \\
\hline G3/G4 & 78 & 69.0 & 35 & 31.0 & & 81 & 61.8 & 50 & 38.2 & \\
\hline Resection margins & & & & & 0.132 & & & & & 0.051 \\
\hline Negative. R0 & 77 & 59.7 & 52 & 40.3 & & 85 & 54.8 & 70 & 45.2 & \\
\hline Positive. R1 & 15 & 78.9 & 4 & 21.1 & & 15 & 78.9 & 4 & 21.1 & \\
\hline UICC & & & & & 0.301 & & & & & 0.047 \\
\hline $\mathrm{I} / \mathrm{II}$ & 34 & 56.7 & 26 & 43.3 & & 41 & 49.4 & 42 & 50.6 & \\
\hline III/IV & 58 & 65.9 & 30 & 34.1 & & 59 & 64.8 & 32 & 35.2 & \\
\hline
\end{tabular}

Bold print denotes $\mathrm{P}<0.05$. SVV, survivin.

(Table V, Fig. 2D). However, multivariate analysis revealed UICC stage, grading and gender as independent prognostic factors for intestinal type GCs (Table VI). Interestingly, high XIAP expression in tumor centers was found to be an independent prognostic factor in both diffuse type and mixed type GCs (Table VI). Other independent prognostic factors for the diffuse and mixed type were UICC stage, patient's age and overexpression of XIAP in the invasion front.

\section{Discussion}

In this study we explored survivin and XIAP expression in a large number of surgically resected GC tissue specimens. In contrast to previously published studies, we specifically analyzed survivin and XIAP expression patterns in distinct compartments (tumor center and invasion front) of the primary tumor, lymph node metastases and non-neoplastic gastric mucosa. This accurate protein expression analysis revealed important results that have not been demonstrated elsewhere.

First and in accordance with previous reports (24,34-36), we found an increased survivin and XIAP expression in GC tissue when compared to adjacent normal mucosa. This is noteworthy, as the basis for targeted therapy is the identification of a cancer-specific and druggable protein. The second observation, that XIAP expression was significantly higher in lymph node metastasis when compared to corresponding primary tumors supports the theory that XIAP not only functions as inhibitor of apoptosis but also plays a distinct role in metastatic tumor disease. This theory was brought up by Mehrotra et al who could demonstrate that intermolecular cooperation between XIAP and survivin promotes tumor cell invasion and metastasis by an activation of cell 
Table IV. Correlation between nuclear SVV expression and clinicopathological factors in gastric cancer.

\begin{tabular}{|c|c|c|c|c|c|c|c|c|c|c|}
\hline & \multicolumn{5}{|c|}{ SVV (nuc.)-center } & \multicolumn{5}{|c|}{ SVV (nuc.)-invasion } \\
\hline & \multicolumn{2}{|c|}{ Low $n=95$} & \multicolumn{2}{|c|}{ High $n=53$} & \multirow[b]{2}{*}{ P-value } & \multicolumn{2}{|c|}{ Low $n=120$} & \multicolumn{2}{|c|}{ High $n=54$} & \multirow[b]{2}{*}{ P-value } \\
\hline & $\mathrm{n}$ & $\%$ & $\mathrm{n}$ & $\%$ & & $\mathrm{n}$ & $\%$ & $\mathrm{n}$ & $\%$ & \\
\hline Age & & & & & 0.306 & & & & & 0.251 \\
\hline$\leq$ Median & 52 & 68.4 & 24 & 31.6 & & 66 & 73.3 & 24 & 26.7 & \\
\hline >Median & 43 & 59.7 & 29 & 40.3 & & 54 & 64.3 & 30 & 35.7 & \\
\hline Sex & & & & & 0.086 & & & & & 0.163 \\
\hline Men & 31 & 75.6 & 10 & 24.4 & & 42 & 76.4 & 13 & 23.6 & \\
\hline Women & 64 & 59.8 & 43 & 40.2 & & 78 & 65.5 & 41 & 34.5 & \\
\hline Lauren & & & & & 0.133 & & & & & 0.005 \\
\hline diffuse & 40 & 72.7 & 15 & 27.3 & & 53 & 84.1 & 10 & 15.9 & \\
\hline intestinal & 33 & 55.0 & 27 & 45.0 & & 42 & 60.0 & 28 & 40.0 & \\
\hline mixed & 22 & 66.7 & 11 & 33.3 & & 25 & 61.0 & 16 & 39.0 & \\
\hline Tumor stage & & & & & 0.393 & & & & & 0.503 \\
\hline $\mathrm{T} 1 / \mathrm{T} 2$ & 48 & 60.8 & 31 & 39.2 & & 70 & 66.7 & 35 & 33.3 & \\
\hline $\mathrm{T} 3 / \mathrm{T} 4$ & 47 & 68.1 & 22 & 31.9 & & 50 & 72.5 & 19 & 27.5 & \\
\hline Lymph nodes & & & & & 0.839 & & & & & 0.101 \\
\hline Negative. N0 & 21 & 61.8 & 13 & 38.2 & & 29 & 59.2 & 20 & 40.8 & \\
\hline Positive. N+ & 74 & 64.9 & 40 & 35.1 & & 91 & 72.8 & 34 & 27.2 & \\
\hline Metastasis & & & & & 1.000 & & & & & 0.778 \\
\hline M0 & 84 & 64.1 & 47 & 35.9 & & 108 & 68.4 & 50 & 31.6 & \\
\hline M1 & 11 & 64.7 & 6 & 35.3 & & 12 & 75.0 & 4 & 25.0 & \\
\hline Grading & & & & & 0.843 & & & & & 0.089 \\
\hline $\mathrm{G} 1 / \mathrm{G} 2$ & 22 & 62.9 & 13 & 37.1 & & 25 & 58.1 & 18 & 41.9 & \\
\hline $\mathrm{G} 3 / \mathrm{G} 4$ & 73 & 64.6 & 40 & 35.4 & & 95 & 72.5 & 36 & 27.5 & \\
\hline Resection margins & & & & & 0.801 & & & & & 0.192 \\
\hline Negative. R0 & 82 & 63.6 & 47 & 36.4 & & 104 & 67.5 & 50 & 32.5 & \\
\hline Positive. R1 & 13 & 68.4 & 6 & 31.6 & & 16 & 80.0 & 4 & 20.0 & \\
\hline UICC & & & & & 0.036 & & & & & 0.074 \\
\hline $\mathrm{I} / \mathrm{II}$ & 32 & 53.3 & 28 & 46.7 & & 51 & 62.2 & 31 & 37.8 & \\
\hline III/IV & 63 & 71.6 & 25 & 28.4 & & 69 & 75.0 & 23 & 25.0 & \\
\hline
\end{tabular}

Bold print denotes $\mathrm{P}<0.05$. SVV, survivin.

motility kinases FAK and Src (11). The positive correlation between cytoplasmic survivin and XIAP expression in GC tissue specimens is the third interesting finding of our analysis. This observation might be explained by evidence suggesting that mitochondrial survivin released into the cytosol has the ability to associate with XIAP (37). This survivin-XIAP complex stabilizes XIAP by protection against ubiquitin-dependent degradation $(11,16,19,38)$.

Our analysis demonstrated that expression of XIAP and survivin was associated with intestinal type and well-differentiated adenocarcinoma, as well as less advanced tumor stages. In accordance with our findings Nakamura et al and Zhu et al discovered not only increased survivin levels in intestinal type GC but also in precancerous lesions. Consequently, they concluded that survivin occurs as an early event in GC development $(39,40)$. In contrast, our recently published meta-analysis investigating the relationship between survivin expression levels and clinicopathological parameters reported a significant correlation between increased survivin expression and GC with lymph node metastasis (21). However, our meta-analysis investigating the relationship between survivin expression and lymph node metastases demonstrated a high heterogeneity among the trials and included also studies reporting increased survivin expression levels in lymph node-negative GCs $(22,34,41-43)$. Consistent with our observation, two previously published studies found also increased XIAP expression levels in intestinal type and well-differentiated GC specimens $(22,24)$. However, in contrast to our results Kim et al (22) discovered increased XIAP expression levels in advanced GC stages.

Although several studies have addressed the relationship between high survivin levels and poor prognosis in 


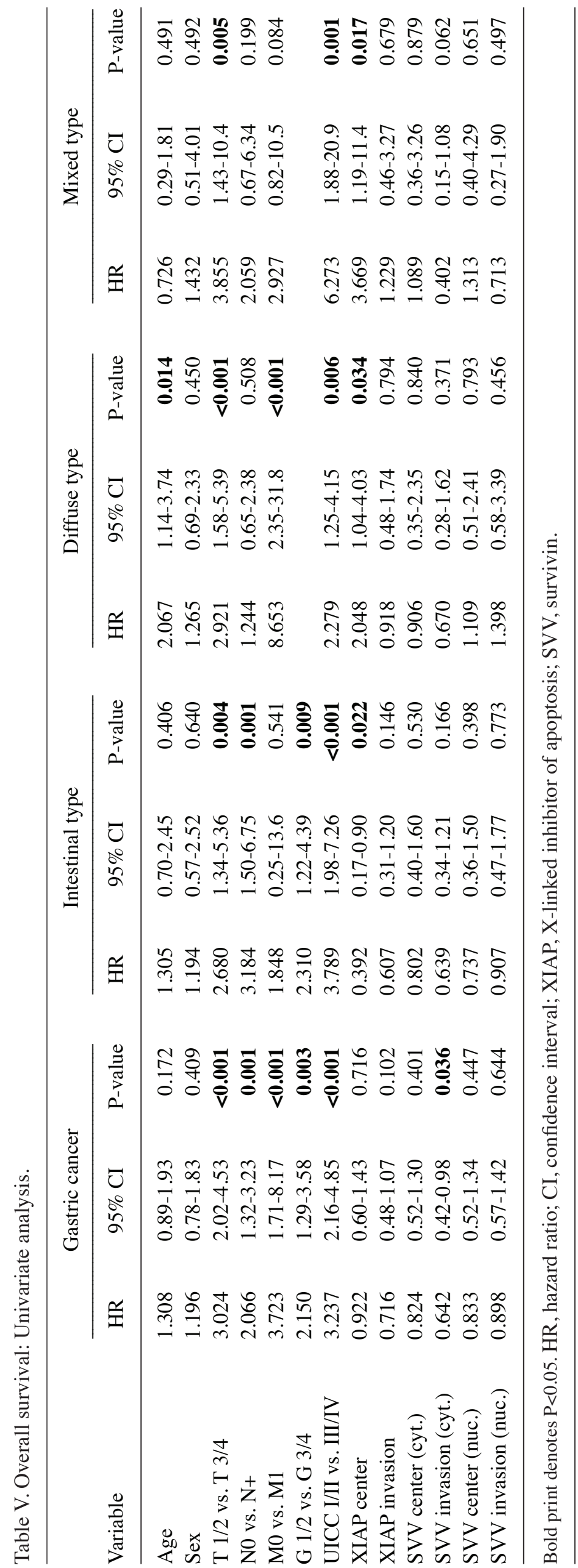



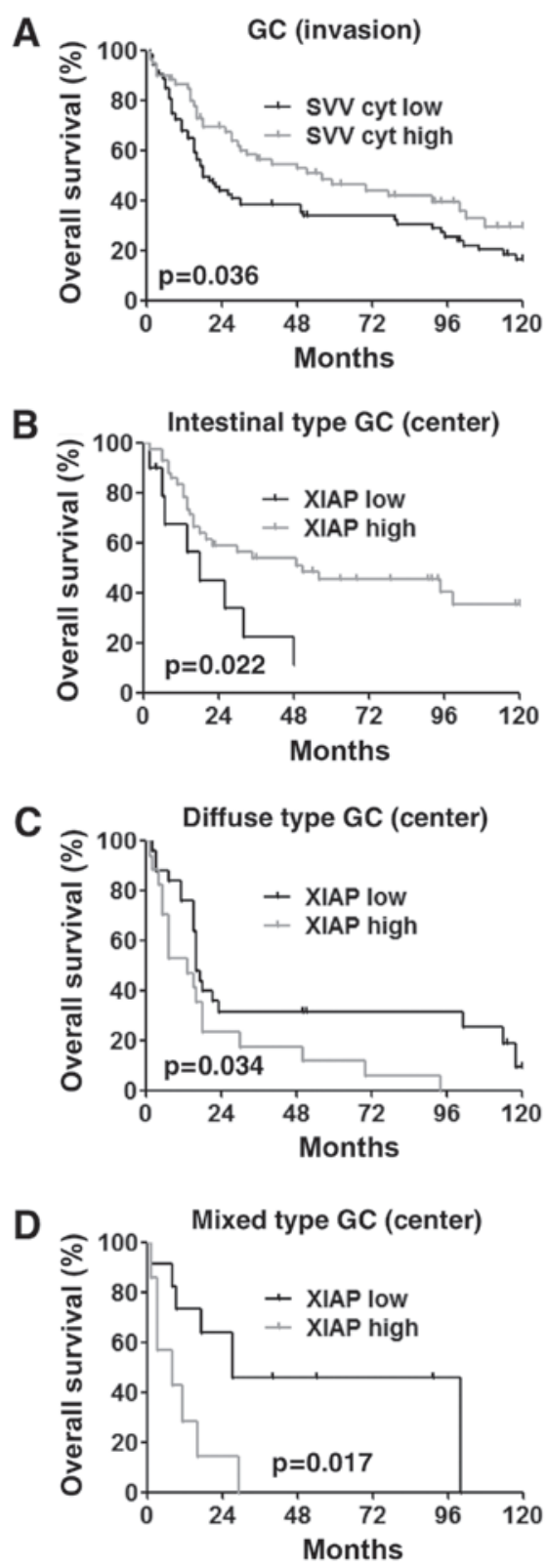

Figure 2. Overall survival of gastric cancer (GC) patients stratified according to survivin (SVV) or X-linked inhibitor of apoptosis (XIAP) expression. GC were categorized into groups of high (IRS $>2$ ) or low (IRS $\leq 2$ ) survivin and XIAP expression. Data were analyzed using the log-rank test. (A) Kaplan Meier curve correlating overall survival with cytoplasmic SVV expression in invasion fronts of all GCs. (B-D) Kaplan Meier curves correlating overall survival with XIAP expression in tumor centers of (B) intestinal type, (C) diffuse type or (D) mixed type GCs.

GC patients $(25-27,35,39,44)$, many conflicting findings exist $(22,34,40,45)$. Of note, our analysis of survivin expression revealed no significant correlation between survivin expression and patients overall survival. The observation that XIAP might serve as a prognostic biomarker in GC was first described by Kim et al (22). Importantly, our study refined these observations by performing a detailed survival analysis with respect to XIAP in the tumor center and invasion front of the different histological subgroups. Accordingly, our data demonstrate that in contrast to intestinal type GCs, high XIAP expression serves as an independent negative prognostic marker for diffuse and mixed type GCs. 
We also observed a tendency towards higher XIAP expression levels in tumors without lymph node or distant metastasis. However, these differences were not statistically significant, but indicate a potential role for XIAP in the early phase of GC development.

Taken together, our survivin and XIAP expression analysis in GC tissue specimens revealed that only XIAP might be regarded as a prognostic marker in diffuse and mixed type GCs. Thus, our results might suggest that XIAP could be one of the key molecular targets especially in the subtype of diffuse GC which is more commonly associated with a very poor prognosis. However, both IAPs represent promising candidates for targeted therapy approaches, as they were significantly overexpressed in the vast majority of all GC specimens investigated. Different strategies to target IAP proteins for cancer therapy have been investigated thus far and, among them, small-molecule IAP antagonists and antisense oligonucleotides have reached the most attention $(14,46,47)$. Currently, the most intensively investigated survivin antagonist is the small imidazolium-based compound YM155 (Sepantronium bromide). This small molecule survivin inhibitor demonstrated impressive anticancer activity in several preclinical studies using cancer cell lines originating from various types of cancer, including GC cells $(30,48-50)$ and was well tolerated in phase I studies $(51,52)$. Concerning the potential efficacy of anti-XIAP treatment strategies in GC initial promising results have been published. Wang et al demonstrated that the XIAP inhibitor Embelin significantly reduced GC cell viability, induced apoptosis, and enhanced 5-FU antitumor activity in vitro (53). Furthermore, Tong et al established that downregulation of XIAP via antisense RNA can induce apoptosis and enhance chemotherapeutic agent sensitivity in GC cells (54). Given these promising findings future studies should address the question whether or not survivin and XIAP directed therapy approaches are able to improve the survival of GC patients.

However, our study might have some limitations. One limitation is the relatively short follow up period and the design as a single-institution retrospective analysis. In addition, we detected survivin by using a polyclonal antibody that might also bind to the different alternative splice variants of survivin. Of note, in previous studies using monoclonal as well as aforementioned polyclonal antibodies, we failed to detect survivin splice variants on protein levels (55). Importantly, the specificity of the antibody that we used in our study was also confirmed in knock down experiments that we recently performed (30).

Despite these limitations, we are the first to demonstrate that high XIAP expression is a negative prognostic factor exclusively in the subgroup of diffuse and mixed type GC. These results are important since they provide a rationale for future studies investigating the therapeutic efficacy of anti-XIAP treatment strategies, especially in patients with diffuse and mixed type GCs.

\section{References}

1. Ferlay J, Soerjomataram I, Ervik M, Dikshit R, Eser S, Mathers C, Rebelo M, Parkin DM, Forman D and Bray F: GLOBOCAN 2012 v1.0, Cancer Incidence and Mortality Worldwide: IARC CancerBase No. 11 (Internet). International Agency for Research on Cancer, Lyon, 2013. http://globocan.iarc.fr. Accessed December 28, 2016 .
2. Howlader N NA, Krapcho M, Miller D, Bishop K, Altekruse SF, Kosary CL, Yu M, Ruhl J, Tatalovich Z, Mariotto A, et al: SEER Cancer Statistics Review, 1975-2013. National Cancer Institute, Bethesda, MD, 2016. http://seercancergov/csr/1975_2013/. Accessed December 28, 2016.

3. D'Angelica M, Gonen M, Brennan MF, Turnbull AD, Bains M and Karpeh MS: Patterns of initial recurrence in completely resected gastric adenocarcinoma. Ann Surg 240: 808-816, 2004.

4. Okines AF, Ashley SE, Cunningham D, Oates J, Turner A, Webb J, Saffery C, Chua YJ and Chau I: Epirubicin, oxaliplatin, and capecitabine with or without panitumumab for advanced esophagogastric cancer: Dose-finding study for the prospective multicenter, randomized, phase II/III REAL-3 trial. J Clin Oncol 28: 3945-3950, 2010

5. Cunningham D, Okines AF and Ashley S: Capecitabine and oxaliplatin for advanced esophagogastric cancer. N Engl J Med 362: 858-859, 2010.

6. Roth AD, Fazio N, Stupp R, Falk S, Bernhard J, Saletti P, Köberle D, Borner MM, Rufibach K, Maibach R, et al: Docetaxel, cisplatin and fluorouracil; docetaxel and cisplatin; and epirubicin, cisplatin and fluorouracil as systemic treatment for advanced gastric carcinoma: A randomized phase II trial of the Swiss Group for Clinical Cancer Research. J Clin Oncol 25: 3217-3223, 2007.

7. Giuliani F, Molica S, Maiello E, Battaglia C, Gebbia V, Di Bisceglie M, Vinciarelli G, Gebbia N and Colucci G; Gruppo Oncologico dell' Italia Meridionale (prot. 2106): Irinotecan (CPT-11) and mitomycin-C (MMC) as second-line therapy in advanced gastric cancer: A phase II study of the Gruppo Oncologico dell' Italia Meridionale (prot. 2106). Am J Clin Oncol 28: 581-585, 2005.

8. Wagner AD and Moehler M: Development of targeted therapies in advanced gastric cancer: Promising exploratory steps in a new era. Curr Opin Oncol 21: 381-385, 2009.

9. Mita AC, Mita MM, Nawrocki ST and Giles FJ: Survivin: Key regulator of mitosis and apoptosis and novel target for cancer therapeutics. Clin Cancer Res 14: 5000-5005, 2008.

10. Srinivasula SM and Ashwell JD: IAPs: What's in a name? Mol Cell 30: 123-135, 2008.

11. Mehrotra S, Languino LR, Raskett CM, Mercurio AM, Dohi T and Altieri DC: IAP regulation of metastasis. Cancer Cell 17: 53-64, 2010.

12. Bertrand MJ, Milutinovic S, Dickson KM, Ho WC, Boudreault A, Durkin J, Gillard JW, Jaquith JB, Morris SJ and Barker PA: cIAP1 and cIAP2 facilitate cancer cell survival by functioning as E3 ligases that promote RIP1 ubiquitination. Mol Cell 30: 689-700, 2008

13. Altieri DC: New wirings in the survivin networks. Oncogene 27: 6276-6284, 2008.

14. Fulda S and Vucic D: Targeting IAP proteins for therapeutic intervention in cancer. Nat Rev Drug Discov 11: 109-124, 2012.

15. Altieri DC: Survivin-The inconvenient IAP. Semin Cell Dev Biol 39: 91-96, 2015.

16. Dohi T, Beltrami E, Wall NR, Plescia J and Altieri DC: Mitochondrial survivin inhibits apoptosis and promotes tumorigenesis. J Clin Invest 114: 1117-1127, 2004.

17. Krieg A, Correa RG, Garrison JB, Le Negrate G, Welsh K, Huang Z, Knoefel WT and Reed JC: XIAP mediates NOD signaling via interaction with RIP2. Proc Natl Acad Sci USA 106: 14524-14529, 2009.

18. Krieg A and Reed JC: IAPs and their emergent role in NLR signaling. Cell Cycle 9: 426-427, 2010.

19. Dohi T, Okada K, Xia F, Wilford CE, Samuel T, Welsh K, Marusawa $\mathrm{H}$, Zou H, Armstrong R, Matsuzawa S, et al: An IAP-IAP complex inhibits apoptosis. J Biol Chem 279: 34087-34090, 2004.

20. Lu M, Lin SC, Huang Y, Kang YJ, Rich R, Lo YC, Myszka D, Han J and Wu H: XIAP induces NF-kappaB activation via the BIR1/TAB1 interaction and BIR1 dimerization. Mol Cell 26: 689-702, 2007.

21. Krieg A, Baseras B, Tomczak M, Verde PE, Stoecklein NH and Knoefel WT: Role of survivin as prognostic and clinicopathological marker in gastric cancer: A meta-analysis. Mol Biol Rep 40: 5501-5511, 2013.

22. Kim MA, Lee HE, Lee HS, Yang HK and Kim WH: Expression of apoptosis-related proteins and its clinical implication in surgically resected gastric carcinoma. Virchows Arch 459: 503-510, 2011.

23. Shibata T, Mahotka C, Wethkamp N, Heikaus S, Gabbert HE and Ramp U: Disturbed expression of the apoptosis regulators XIAP, $\mathrm{XAF} 1$, and Smac/DIABLO in gastric adenocarcinomas. Diagn Mol Pathol 16: 1-8, 2007. 
24. Shibata T, Noguchi T, Takeno S, Gabbert HE, Ramp U and Kawahara K: Disturbed XIAP and XAF1 expression balance is an independent prognostic factor in gastric adenocarcinomas. Ann Surg Oncol 15: 3579-3587, 2008.

25. Lee GH, Joo YE, Koh YS, Chung IJ, Park YK, Lee JH, Kim HS, Choi SK, Rew JS, Park CS and Kim SJ: Expression of survivin in gastric cancer and its relationship with tumor angiogenesis. Eur J Gastroenterol Hepatol 18: 957-963, 2006.

26. Song KY, Jung CK, Park WS and Park CH: Expression of the antiapoptosis gene Survivin predicts poor prognosis of stage III gastric adenocarcinoma. Jpn J Clin Oncol 39: 290-296, 2009.

27. Meng JR, Tang HZ, Zhou KZ, Shen WH and Guo HY: TFF3 and survivin expressions associate with a lower survival rate in gastric cancer. Clin Exp Med 13: 297-303, 2013

28. McShane LM, Altman DG, Sauerbrei W, Taube SE, Gion M and Clark GM; Statistics Subcommittee of the NCI-EORTC Working Group on Cancer Diagnostics: REporting recommendations for tumour MARKer prognostic studies (REMARK). Br J Cancer 93: 387-391, 2005.

29. International Union Against Cancer: TNM classification of malignant tumours. Sobin LH and GM WC (eds). 7th edition. Wiley-Liss, New York, NY, 2009.

30. Dizdar L, Oesterwind KA, Riemer JC, Werner TA, Mersch S, Möhlendick B, Schütte SC, Verde PE, Raba K, Topp SA, et al Preclinical assesement of survivin and XIAP as prognostic biomarkers and therapeutic targets in gastroenteropancreatic neuroendocrine neoplasia. Oncotarget 8: 8369-8382, 2017.

31. Remmele W and Stegner HE: Recommendation for uniform definition of an immunoreactive score (IRS) for immunohistochemical estrogen receptor detection (ER-ICA) in breast cancer tissue. Pathologe 8: 138-140, 1987 (In German).

32. Werner TA, Tamkan-Ölcek Y, Dizdar L, Riemer JC, Wolf A, Cupisti K, Verde PE, Knoefel WT and Krieg A: Survivin and XIAP: Two valuable biomarkers in medullary thyroid carcinoma. Br J Cancer 114: 427-434, 2016.

33. Burnham KP, Anderson DR and Burnham KP (eds): Model Selection And Multimodel Inference: A Practical Information-Theoretic Approach. 2nd edition. Springer, New York, NY, 2002.

34. Lu CD, Altieri DC and Tanigawa N: Expression of a novel antiapoptosis gene, survivin, correlated with tumor cell apoptosis and p53 accumulation in gastric carcinomas. Cancer Res 58 1808-1812, 1998

35. Meng H, Lu C, Mabuchi $\mathrm{H}$ and Tanigawa N: Prognostic significance and different properties of survivin splicing variants in gastric cancer. Cancer Lett 216: 147-155, 2004

36. Da CL, Xin Y, Zhao J and Luo XD: Significance and relationship between Yes-associated protein and survivin expression in gastric carcinoma and precancerous lesions. World J Gastroenterol 15: 4055-4061, 2009.

37. Dohi T, Xia F and Altieri DC: Compartmentalized phosphorylation of IAP by protein kinase A regulates cytoprotection. Mol Cell 27: 17-28, 2007.

38. Arora V, Cheung $\mathrm{HH}$, Plenchette S, Micali OC, Liston P and Korneluk RG: Degradation of survivin by the X-linked inhibitor of apoptosis (XIAP)-XAF1 complex. J Biol Chem 282. 26202-26209, 2007

39. Nakamura M, Tsuji N, Asanuma K, Kobayashi D, Yagihashi A, Hirata K, Torigoe T, Sato N and Watanabe N: Survivin as a predictor of cis-diamminedichloroplatinum sensitivity in gastric cancer patients. Cancer Sci 95: 44-51, 2004.

40. Zhu XD, Lin GJ, Qian LP and Chen ZQ: Expression of survivin in human gastric carcinoma and gastric carcinoma model of rats. World J Gastroenterol 9: 1435-1438, 2003.
41. Deng H, Wu RL, Zhou HY, Huang X, Chen Y and Liu LJ: Significance of Survivin and PTEN expression in full lymph node-examined gastric cancer. World J Gastroenterol 12: 1013-1017, 2006

42. Okayama H, Kumamoto K, Saitou K, Hayase S, Kofunato Y, Sato Y, Miyamoto K, Nakamura I, Ohki S, Sekikawa K and Takenoshita S: CD44v6, MMP-7 and nuclear Cdx2 are significant biomarkers for prediction of lymph node metastasis in primary gastric cancer. Oncol Rep 22: 745-755, 2009.

43. Tsuburaya A, Noguchi Y, Yoshikawa T, Saito A, Doi C, Okamoto T and Fukuzawa K: An anti-apoptosis gene, survivin and telomerase expression in gastric cancer. Hepatogastroenterology 49: $1150-1152,2002$

44. Bury J, Szumiło J, Dąbrowski A, Ciechański A, Śliwińska J and Wallner G: Vascular endothelial growth factor and survivin immunostaining in gastric adenocarcinoma. Pol Przegl Chir 84: 341-347, 2012.

45. Okada E, Murai Y, Matsui K, Isizawa S, Cheng C, Masuda M and Takano Y: Survivin expression in tumor cell nuclei is predictive of a favorable prognosis in gastric cancer patients. Cancer Lett 163: 109-116, 2001.

46. Ndubaku C, Cohen F, Varfolomeev E and Vucic D: Targeting inhibitor of apoptosis proteins for therapeutic intervention. Future Med Chem 1: 1509-1525, 2009.

47. LaCasse EC, Mahoney DJ, Cheung HH, Plenchette S, Baird S and Korneluk RG: IAP-targeted therapies for cancer. Oncogene 27: 6252-6275, 2008

48. Nakahara T, Kita A, Yamanaka K, Mori M, Amino N, Takeuchi M, Tominaga F, Hatakeyama S, Kinoyama I, Matsuhisa A, et al: YM155, a novel small-molecule survivin suppressant, induces regression of established human hormone-refractory prostate tumor xenografts. Cancer Res 67: 8014-8021, 2007.

49. Cheng XJ, Lin JC, Ding YF, Zhu L, Ye J and Tu SP: Survivin inhibitor YM155 suppresses gastric cancer xenograft growth in mice without affecting normal tissues. Oncotarget 7: 7096-7109, 2016.

50. Ghadimi MP, Young ED, Belousov R, Zhang Y, Lopez G, Lusby K, Kivlin C, Demicco EG, Creighton CJ, Lazar AJ, et al: Survivin is a viable target for the treatment of malignant peripheral nerve sheath tumors. Clin Cancer Res 18: 2545-2557, 2012.

51. Satoh T, Okamoto I, Miyazaki M, Morinaga R, Tsuya A, Hasegawa Y, Terashima M, Ueda S, Fukuoka M, Ariyoshi Y, et al: Phase I study of YM155, a novel survivin suppressant, in patients with advanced solid tumors. Clin Cancer Res 15: 3872-3880, 2009.

52. Tolcher AW, Mita A, Lewis LD, Garrett CR, Till E, Daud AI, Patnaik A, Papadopoulos K, Takimoto C, Bartels P, et al: Phase I and pharmacokinetic study of YM155, a small-molecule inhibitor of survivin. J Clin Oncol 26: 5198-5203, 2008.

53. Wang DG, Sun YB, Ye F, Li W, Kharbuja P, Gao L, Zhang DY and Suo J: Anti-tumor activity of the X-linked inhibitor of apoptosis (XIAP) inhibitor embelin in gastric cancer cells. Mol Cell Biochem 386: 143-152, 2014

54. Tong QS, Zheng LD, Wang L, Zeng FQ, Chen FM, Dong JH and Lu GC: Downregulation of XIAP expression induces apoptosis and enhances chemotherapeutic sensitivity in human gastric cancer cells. Cancer Gene Ther 12: 509-514, 2005.

55. Mahotka C, Krieg T, Krieg A, Wenzel M, Suschek CV, Heydthausen M, Gabbert HE and Gerharz CD: Distinct in vivo expression patterns of survivin splice variants in renal cell carcinomas. Int J Cancer 100: 30-36, 2002. 